\author{
Einführung in das Schwerpunktthema
}

\title{
Vom Weltmarkt zum Wochenmarkt
}

D

Von Guido Nischwitz und Reimar Molitor nährungswirtschaft am Leitbild der Nachhaltigen Entwicklung ist mittlerweile in Politik, Gesellschaft, Wissenschaft und Wirtschaft fast unumstritten. Auf verschiedenen räumlichen Ebenen (global, $\mathrm{EU}$, Bund, Regionen) setzen die Akteure zur Umsetzung an. Über den kleinsten gemeinsamen Nenner Nachhaltigkeit hinaus zeigen sich allerdings erhebliche Unterschiede. In Abhängigkeit von Ausgangsbedingungen und spezifischen Interessen der Akteure weichen die Definitionen von Nachhaltigkeit, die Formulierung von Aufgabenschwerpunkten und Zielen und sowie die Umsetzungsbemühungen deutlich voneinander $a b$.

Im globalen Kontext hat die Land- und Ernährungswirtschaft weiterhin eine große sozioökonomische Bedeutung. Dementsprechend werden bei der laufenden Verhandlungsrunde der Welthandelsorganisation (WTO), den EU-Beitrittsgesprächen oder im Agenda 21-Dokument der RioKonferenz der nachhaltigen Steigerung der Nahrungsmittelproduktion und Verbesserung der Ernährungssituation ein hoher Stellenwert zugebilligt (1). Eng damit verbunden ist die Sicherung von Beschäftigung, betrieblichen Existenzen und Einkommen.

In der EU wird angesichts des anhaltenden wirtschaftlichen Bedeutungsverlustes die sogenannte Multifunktionalität der Landwirtschaft neu herausgestellt. Neben der Sicherung einer qualitativ hochwertigen Nahrungsmittelversorgung wird dabei ihr Beitrag für den Umwelt- und Naturschutz, zur soziokulturellen Stabilisierung ländlicher Räume sowie zur Leistung spezifischer Dienstleistungen hervorgehoben.

Die bundesdeutsche Debatte um Nachhaltigkeit in der Land- und Ernährungswirtschaft wird durch folgende Themenstellungen bestimmt: Integration von Umwelt- und Naturschutzaspekten, Bestimmung nachhaltiger Produktionsweisen, Auswahl von Agrar-Umweltindikatoren, Bedeutung des regional-ökologischen Ansatzes, nachhaltiger Konsum, Handlungsbedarf bei den politisch-rechtlichen Rahmenbedingungen, Übertragbarkeit von Praxiserfahrungen. Beispielsweise versucht im Rahmen einer kontroversen Diskussion die traditionelle landwirtschaftliche Interessenvertretung die vorherrschenden Bewirtschaftungsformen (,gute fachliche Praxis") mit nachhaltigem Wirtschaften gleichzusetzen.

\section{- Ziele nachhaltiger Land- und Ernährungswirtschaft}

Fast 90 Prozent aller Nahrungsmittel werden im Rahmen eines mehrstufigen Absatzweges über das Ernährungsgewerbe, Gemeinschaftsverpflegungseinrichtungen und den Lebensmittel-Einzelhandel vermarktet. Dennoch spielt die Ernährungswirtschaft in der Nachhaltigkeitsdebatte nur eine untergeordnete Rolle.

Für eine nachhaltige Nahrungsmittelverarbeitung und -vermarktung lassen sich als Ziele auffuihren:

- Verringerung des Transportaufwandes,

- Senkung des Ressourcenverbrauches (vor allem Wasser, Energie, Verpackung),

- Stärkung der lokalen/ regionalen Erfassung, Verarbeitung, Vermarktung und Entsorgung,

- Sicherstellung einer nachvollziehbaren Qualitäts- und Herkunftssicherung,

- Versorgung der Bevölkerung mit qualitativ hochwertigen Nahrungsmitteln,

- Angebot von fair gehandelten und ökologisch produzierten Nahrungsmitteln,

- Berücksichtigung von Verbraucherinteressen, - Leistung eines Beitrages zur gesundheits- und umweltorientierten Ernährung (2).

Die erhöhte öffentliche Aufmerksamkeit für den landwirtschaftlichen Bereich lässt sich auf dessen spezifische Raumbindung und die damit einhergehende Abhängigkeit und Nutzung von natürlichen Ressourcen zurückführen. Neben den ökologischen Auswirkungen und Leistungen der Landwirtschaft rücken im Zuge der Nachhaltigkeitsdebatte verstärkt ökonomische und soziale Fragestellungen in den Mittelpunkt. Zusammengefasst lassen sich folgende Zielsetzungen und Schwerpunkte für eine nachhaltige Landwirtschaft herausfiltern:

- langfristige Sicherung der Nahrungsmittelversorgung und -qualität,

- Schonung nicht erneuerbarer Ressourcen,

- effiziente Nutzung und Schutz der erneuerbaren Ressourcen,

- Erhaltung und Nutzung der biologischen Vielfalt,

- artgerechte Tierhaltung,
- Sicherstellung der ökonomischen Existenzfähigkeit landwirtschaftlicher Betriebe,

- Förderung von ökologischen und sozialen Dienstleistungen,

- Aufrechterhaltung einer weitgehend flächendeckenden Landbewirtschaftung (3).

Zahlreiche Experten sehen diese Zielsetzungen am ehesten durch eine Land- und Ernährungswirtschaft erfüllt, die sich an der Schließung regionaler Wirtschafts- und Stoffkreisläufe und am ökologischen Landbau orientiert.

\section{- Wachsender Handlungsbedarf}

Die dynamischen Veränderungen der wirtschaftlichen und politisch-rechtlichen Rahmenbedingungen spielen sich in einem Spannungsfeld von Globalisierungs- und Regionalisierungstendenzen ab. Auf der einen Seite kommt es durch die Handelsliberalisierung zu einem Angebots- und Preisdruck, auf der anderen Seite ergeben sich aufgrund der Aufwertung der regionalen Ebene neue Entwicklungschancen. Gleichzeitig steigen die Anforderungen der Gesellschaft an eine nachhaltige Wirtschaftsweise

Die voranschreitende Liberalisierung des Weltagrarhandels wird in diesem Zusammenhang erhebliche Auswirkungen auf die künftige Gestaltung der EU-Agrarpolitik und den agrarstrukturellen Wandel haben. Als weltgrößter Agrarexporteur (Marktanteil 38 Prozent) mit einem Anteil von 90 Prozent der global gezahlten Exportsubventionen steht vor allem die EU bei der neuen WTO-Runde unter Handlungsdruck. Zur Disposition steht das Grundgerüst der gemeinsamen Agrarpolitik, bei der bislang Preissenkungen durch flächengebundene direkte Einkommensübertragungen kompensiert werden. Diese Maßnahmen dürften allerdings in der WTO kaum als GATT-konform (,green-box-fähig“) eingestuft werden. Stattdessen sieht sich die EU mit Forderungen nach einer Reduzierung der Exportsubventionen, einer stärkeren Öffnung ihrer Märkte und weiteren Preissenkungen ohne Ausgleichsleistungen konfrontiert. Eine Lösung könnte die Verknüpfung staatlicher Zahlungen mit definierten Umweltleistungen sein. Dies würde auch einen wichtigen Schritt zur Umsetzung einer nachhaltigen Entwicklung beinhalten. Die Chance zur Einführung einer solchen Maßnahme wurde allerdings von der EU beim Reformpaket Agenda 2000 nicht genutzt.

Die anstehende Osterweiterung der EU erfordert gleichfalls eine umfassende Veränderung der Agrarpolitik in den Bereichen Marktordnung und 
Strukturpolitik. Die vorrangig am Ziel der Produktivitätssteigerung orientierte EU-Agrarpolitik und Landbewirtschaftung führte bislang zu einer regionalen und betrieblichen Intensivierung, Spezialisierung und Rationalisierung. Die damit verbundenen Beeinträchtigungen der natürlichen Ressourcen sind vielfach untersucht und mittlerweile unbestritten. Obwohl sich die EU im Vertrag von Amsterdam und bei der Agenda 2000 zur Umsetzung einer nachhaltigen Entwicklung verpflichtet hat, ist bislang eine grundlegend neue Ausrichtung der Agrarpolitik ausgeblieben.

\section{Wie geht es weiter?}

Die Liberalisierung des Weltagrarhandels, die EU-Osterweiterung und die aktuelle EU-Agrarpolitik wird zu einer Beschleunigung des Strukturwandels führen. Insbesondere aus standortbedingt benachteiligten Regionen wird sich die landwirtschaftliche Erzeugung weiter zurückziehen. Dieser Entwicklungstrend lässt sich seit geraumer Zeit in kleinbetrieblich strukturierten Regionen Süddeutschlands, in Mittelgebirgslagen oder in Teilregionen Ostdeutschlands beobachten. Die wachsende Aufspaltung der Kulturlandschaft in intensiv bewirtschaftete und in brachfallende Flächen und Regionen wirft hier bereits erhebliche soziokulturelle, ökologische und wirtschaftliche Probleme auf.

Um diesem Entwicklungsprozess entgegenwirken zu können, bedarf es innovativer und regional angepasster Entwicklungskonzepte und Maßnahmen. Von den Betroffenen vor Ort wird zunehmend das Konzept der Nachhaltigkeit aufgegriffen, um neue Entwicklungschancen für die Landund Ernährungswirtschaft und damit für ihre Region zu erarbeiten. Im Rahmen von integrierten Entwicklungskonzepten stehen neben der Stärkung der regionalen Wirtschaft auch soziale und ökologische Aspekte im Vordergrund. Mit der Forcierung der Direkt- und Regionalvermarktung, die mit einer umweltverträglichen und tierartgerechten Produktion sowie spezifischen Dienstleistungen verknüpft wird, eröffnen sich neue Entwicklungsperspektiven.

Die bisher aufgearbeiteten regionalen Ansätze und Umsetzungsschritte erweisen sich nicht nur für den ländlichen Raum als beispielgebend. Verstärkt nehmen sich auch Akteure im StadtUmland-Geflecht und in Ballungsräumen dieser Thematik an.

Für eine erfolgreiche Umsetzung einer nachhaltigen Land- und Ernährungswirtschaft stellt die regionale Ebene derzeit die Arena. Die Erfahrun- gen sind allerdings nur begrenzt übertragbar. Eine wirklich umfassende nachhaltige Entwicklung kann nur gelingen, wenn von den Akteuren auf allen politisch-administrativen, wirtschaftlichen und räumlichen Ebenen Veränderungsprozesse eingeleitet werden. Ein zentraler Schritt hierfür wäre die grundlegende Überarbeitung der bislang eher hemmend wirkenden politischrechtlichen Rahmenbedingungen.

\section{- Die Beiträge im Einzelnen}

Die Beiträge des Schwerpunktes spannen den Bogen zwischen den übergreifenden Rahmenbedingungen einer nachhaltigen Land- und Ernährungswirtschaft und der konkreten Ausgestaltung vor Ort. Während Siegfried Bauer und Guido Nischwitz die politisch-rechtlichen und wirtschaftlichen Rahmenbedingungen beleuchten, bewertet Bernhard Burdick den Weg in Richtung Nachhaltigkeit seit der Veröffentlichung der Studie „Zukunftsfähiges Deutschland“. Ein konkretes Konfliktfeld im Verhältnis von Landwirtschaft und Naturschutz behandelt Jens-Peter Abresch. Die Beiträge von Reimar Molitor sowie Anke Witzel und Martin Tischer beschäftigen sich aus unterschiedlichen Blickwinkeln mit den Erfahrungen auf der regionalen Ebene. Spezifische Ausgangsbedingungen, Chancen und Probleme einer Landwirtschaft in Großstadtnähe stehen schließlich im Mittelpunkt des Artikels von Thomas Fiebig.

\section{Anmerkungen}

(1) Frankfurter Rundschau am 27.11.1999: Sprengsatz für Welthandelskonferenz in Seattle liegt auf den Äckern. (2) Vgl. Umweltbundesamt (UBA) (Hrsg.): Nachhaltiges Deutschland. Berlin 1997. Hagemann, H./ Simshäuser, U.: Instrumente und Strategien zur Förderung des nachhaltigen Konsums - Evaluation und Optimierung. Unveröffentlichter Abschlussbericht des IÖW für das UBA, 2000.

(3) Vgl. UBA a.a.0.; SRU (Hrsg.): Umweltgutachten 1994, Stuttgart 1994; Enquete Kommission "Schutz der Erdatmosphäre": Schutz der grünen Erde, Bonn 1994; IUL (Hrsg.): Nachhaltige Landwirtschaft, 1999; Linck, G./Sprich, H./ Flaig, H./Mohr H.: Nachhaltige Land- und Forstwirtschaft, Berlin 1997. 
(c) 20I0 Authors; licensee IÖW and oekom verlag. This is an article distributed under the terms of the Creative Commons Attribution Non-Commercial No Derivates License (http://creativecommons.org/licenses/by-nc-nd/3.o/), which permits unrestricted use, distribution, and reproduction in any medium, provided the original work is properly cited. 